\title{
Comparison of outcomes in emergency department patients with suspected cardiac chest pain: two-centre prospective observational study in Southern China
}

\author{
Huilin Jiang ${ }^{1}$, Yunmei $\mathrm{Li}^{1}$, Junrong Mo ${ }^{1}$, Xiaohui Chen ${ }^{1}$, Min Li ${ }^{1}$, Peiyi Lin ${ }^{1}$, Kevin K. C. Hung ${ }^{2}$,
} Timothy H. Rainer ${ }^{2}$ and Colin A. Graham ${ }^{2 *}$

\begin{abstract}
Background: Hong Kong (HK) and Guangzhou (GZ) are cities in China with different healthcare systems. This study aimed to compare 30-day and 6-month mortality and characteristics of patients with suspected cardiac chest pain admitted to two emergency departments (ED) in HK and GZ.

Methods: A prospective observational study enrolled patients with suspected cardiac chest pain presenting to EDs in the Prince of Wales Hospital (PWH), HK and the Second Affiliated Hospital of Guangzhou Medical University (AHGZMU),GZ. The primary outcome was 30-day and 6-month mortality.

Results: In total, 996 patients were recruited, 407 cases from GZ and 589 cases from HK.The 30-day and 6-month mortality of chest patients were $3.7 \%$ and $4.7 \%$ in GZand $0.3 \%$ and $1.9 \%$ in HK, respectively. Serum creatinine level (Cr) was an independent factor for 30-day mortality whilst $\mathrm{Cr}$ and systolic blood pressure (SBP) were independent factors for 6-month mortality. In Cox regression analysis, unadjusted and adjusted hazard ratios for 30-day and 6-month mortality in GZ were significantly increased.

Conclusion: The 30-day and 6-month mortality of patients with suspected cardiac chest pain in Guangzhou were higher than in Hong Kong due to due to different baseline clinical characteristics of patients and different distributions of diagnoses, which were associated with different healthcare systems. Serum creatinine and SBP were independent factors for 30-day and 6-month mortality.
\end{abstract}

Keywords: Acute coronary syndrome, Risk stratification, Emergency department, Chest pain, Hong Kong, Guangzhou

\section{Backgroud}

Chest pain is a common chief complaint of patients presenting to emergency departments (ED) globally, and it places a huge burden on ED services [1-3]. The challenge for clinicians is the dual danger of discharging patients at potential high risk and the clinical pressure of EDs crowded with low risk patients. In order to improve the quality of care and survival rates of cardiac patients,

\footnotetext{
* Correspondence: cagraham@cuhk.edu.hk

${ }^{2}$ Accident and Emergency Medicine Academic Unit, Chinese University of Hong Kong, Prince of Wales Hospital, Main Clinical Block and Trauma Centre, Shatin, N.T, Hong Kong, China

Full list of author information is available at the end of the article
}

there is a worldwide impetus to develop and improve systems for emergency cardiac care [4]. Current guidelines emphasize rapid determination of the likelihood that the patient's clinical presentation represents high risk chest pain, such as acute coronaryartery disease, and rapid assessment of the immediate risk for a major adverse cardiac event (MACE) [5]. Hong Kong (HK) and Guangzhou (GZ) are cities in southern China with almost the same ethnicity but different healthcare systems [6]. The healthcare system of HK has adopted health care financing and organizational health systems that are commonly seen in centrally planned economies. In contrast, mainland China has integrated many features of health care systems associated with market economies, whereas both have advanced 
systems of chest pain care in EDs [7-9]. So the healthcare systems of the two cities are quite different under the "one country, two systems" policy [10]. No study has compared clinical outcomes of susceptive cardiac chest pain patients with similar ethnicity under different healthcare systems, and the influence factor of its outcome. Our previous study has compared the clinical ED management and one-year mortality of ST-segment elevation myocardial infarction (STEMI) patients between the two hospitals in HK and GZ [11]. After that, the comparable registries of chest pain database have been developed in Guangzhou and Hong Kong. The objective of the study was to compare 30-day and 6-month outcomes of patients with suspected cardiac chest pain presenting to two EDs in HK and GZ, and contributory factors of outcomes.

\section{Methods}

\section{Study design and setting}

A prospective cohort study conducted from May 2012 to August 2013 in two teaching hospitals.

The Second Affiliated Hospital of Guangzhou Medical University (AHGZMU)is an academic hospital with 1500 beds affiliated with the Guangzhou Medical University and serves a population of approximately 1.56 million people in the Hai Zhu district, Guangzhou. The ED receives more than 150,000 new patients per annum and serves a local population of approximately 1,680,000 people. The Prince of Wales Hospital (PWH) is located in the New Territories in Hong Kong. It is a university hospital with 1400 beds. It sees approximately 150,000 new ED patients per annum and serves a local population of approximately 800,000 people.

\section{Inclusion and exclusion criteria}

Consecutive ED patient $\geq 18$ years old, with a chief complaint of chest pain or discomfort were recruited. Patients were excluded if they clearly had a non-cardiac cause of chest pain. Confirmed STEMI patients at ED presentation were also excluded as they did not have undifferentiated chest pain. Patients were excluded if they were unable or unwilling to provide informed consent or unable to be contacted after discharge.

\section{Measurements and data collection}

Baseline patient characteristics, the characteristics of the chest pain (such as location, feature and radiation), vital signs, medical history, family history of coronary artery disease (CAD), ECG results and contact information to facilitate subsequent follow-up were collected and recorded in a computerized database. The HEART score was recorded and calculated by research staff. The details of the HEART score are shown in Additional file 1. Guidelines recommend that patients with possible ACS but with a normal initial ECG and cardiac markers should be observed in a chest pain unit, where continuous cardiac monitoring and repeated measurement of cardiac markers is available [12]. Point-of care troponin testswere used in GZ, whilst high sensitivity troponin tests were used in HK. Therefore, the results of troponin were divided into four groups $(\leq 1 \times$ normal range, $1 \times-2 \times$ normal range, $2 \times-3 \times$ normal range and $\geq 3 \times$ normal range) for analysis. STdepression on initialECG means ST depression $\geq 1.0 \mathrm{mv}$ in any lead of the initial ECG. The onset-ED time and EDECG time were recorded. Follow-up data were obtained from the Clinical Management System (CMS) in HK and Health Insurance Information Management System (HIIMS) in GZ.

\section{Follow up}

Subsequent visits to ED, hospital readmission for evaluation of chest pain and all cardiac procedures performed were retrieved from CMS in HK and HIIMS in GZ, and verified via telephone at 30-days and 6-months followup after initial presentation. Furthermore, death, myocardial infarction, readmission for ACS, and all cardiac testing and coronary revascularization procedures were also obtained via CMS and HIIMS.

\section{Sample size calculation}

According to our previous study [7], the 30-day MACE rates in Chinese patients presenting to ED with cardiac chest pain could have been as high as the upper limit of the $95 \%$ confidence interval in the low-risk group i.e. $13 \%$, and as low as the lower limit of the $95 \%$ confidence interval in the high-risk group i.e. $23 \%$. To achieve adequate power to address the objectives by using 2-tailed alpha of 0.05 and a power of $80 \%$, the minimum sample size required per group is 220 . We aimed to recruit an extra $30 \%$ in case of unforeseen circumstances and thus at least $230(230 \times 1.3=299)$ patients were required per group. Therefore, the minimum sample size in this study was $598(299 \times 2$ recruiting sites $=598)$.

\section{Outcomes}

The primary outcomefor each patient wasall cause mortality within 30 days and 6 monthsafter initial ED presentation. The secondary outcomes were the contributory factors of primary outcomes.

\section{Statistical analyses}

Continuous variables were presented as median and interquartile range or mean \pm standard deviation as appropriate. Categorical variables were expressed as frequencies. Statistical analysis was performed using SPSS v17.0 (SPSS Inc., IL, USA) and Medcalc v9.5 (MedCalc Software, Mariakerke, Belgium). Summary statistics were used to describe patient characteristics from the Hong Kong and Guangzhou groups. Chi-square analysis was used for categorical variables, whilst independent t-tests 
or Mann-Whitney U-tests were used for comparing data from continuous variables. An initial univariate analysis was performed on all variables with 30-day and 6-month mortalityas the dependent variables and presented as unadjusted odds ratios (OR) and 95\% confidence intervals $(95 \% \mathrm{CI})$. Variables with $p \leq 0.1$ were entered into a multivariate ordinal logistic regression. Cumulative survival curves in relation to different hospitals were determined according to the Kaplan-Meier method with the use of log-rank tests for statistical assessment. Cox regression analysis was used to calculate unadjusted and adjusted hazard ratios (HRs) and 95\% CIs for mortality rates after 30 days and 6 months in relation to different hospitals. A $P$ value of $<0.05$ was considered statistically significant and all probabilities were two tailed.

\section{Results}

\section{Recruitment of patients from the two centers}

Patients were recruited from 17 March 2012 to $14 \mathrm{Au}$ gust 2013. The flow chart of the patient recruitment was shown in Fig. 1. There were 1025 eligible patients, with 589 cases in HK and 436 cases in GZ. Twenty-nine patients were excluded due to lack of follow-up data, leaving 996 patients (407 cases from GZ and 589 cases from HK) in the study. The mean age of the 996 patients was $65.1 \pm 14.5$ years and $55.3 \%$ were males.

\section{Baseline characteristics and final diagnoses of chest pain patients}

Table 1 shows the characteristics of chest pain patients from GZ and HK. Compared to patients in HK, patients in
GZ were older and had lower proportions of past history of diabetes, hyperlipidaemia and chronic heart failure, lower proportion of NHYA class I and lower blood pressure. Troponin is a key biomarker to detect myocardial injury in the two hospitals. Troponin was used as a myocardial injury biomarker in 98\% patients in $\mathrm{HK}$ and $70 \%$ patients in GZ. Creatine kinase $(\mathrm{CK})$ and creatine kinase isoenzyme-MB (CK-MB) were the predominant biomarkers used in GZ, in which $98 \%$ patients had a CK level determined. However, these tests were rarely used in HK. Ischaemic cardiac chest pain was diagnosed in 120 (29.4\%) patients in GZ $(9.8 \%$ with NSTEMI, $16.0 \%$ with unstable angina and $3.7 \%$ with stable angina). Non-ischaemic cardiac aetiologies and noncardiac aetiologies were found in $136(33.4 \%)$ and 151 (37.1\%) patients respectively. In HK, 163 (27.7\%) patients had ischaemic cardiac chest pain, including 5.4\% with NSTEMI, $12.1 \%$ with unstable angina and $10.2 \%$ with stable angina. $84(14.3 \%)$ patients were diagnosed as having non-ischaemic cardiac aetiologies and 342 (58.1\%) were diagnosed with non-cardiac aetiologies. There were significant differences in the number of cases diagnosed with non-ischaemic cardiac aetiologies and non-cardiac chest pain between $\mathrm{GZ}$ and $\mathrm{HK}(P<0.0001)$, whilst there was no significant difference in the number of cases diagnosed with ischaemic cardiac chest pain between the two hospitals.There were no significant differences in the number of readmission of 30-day and 6-month.

\section{Comparison of clinical outcomes of chest pain patients in the two centres}

Table 2 shows the 30-day and 6-month mortality of chest painpatients. The 30-day mortality rates for ischaemia,

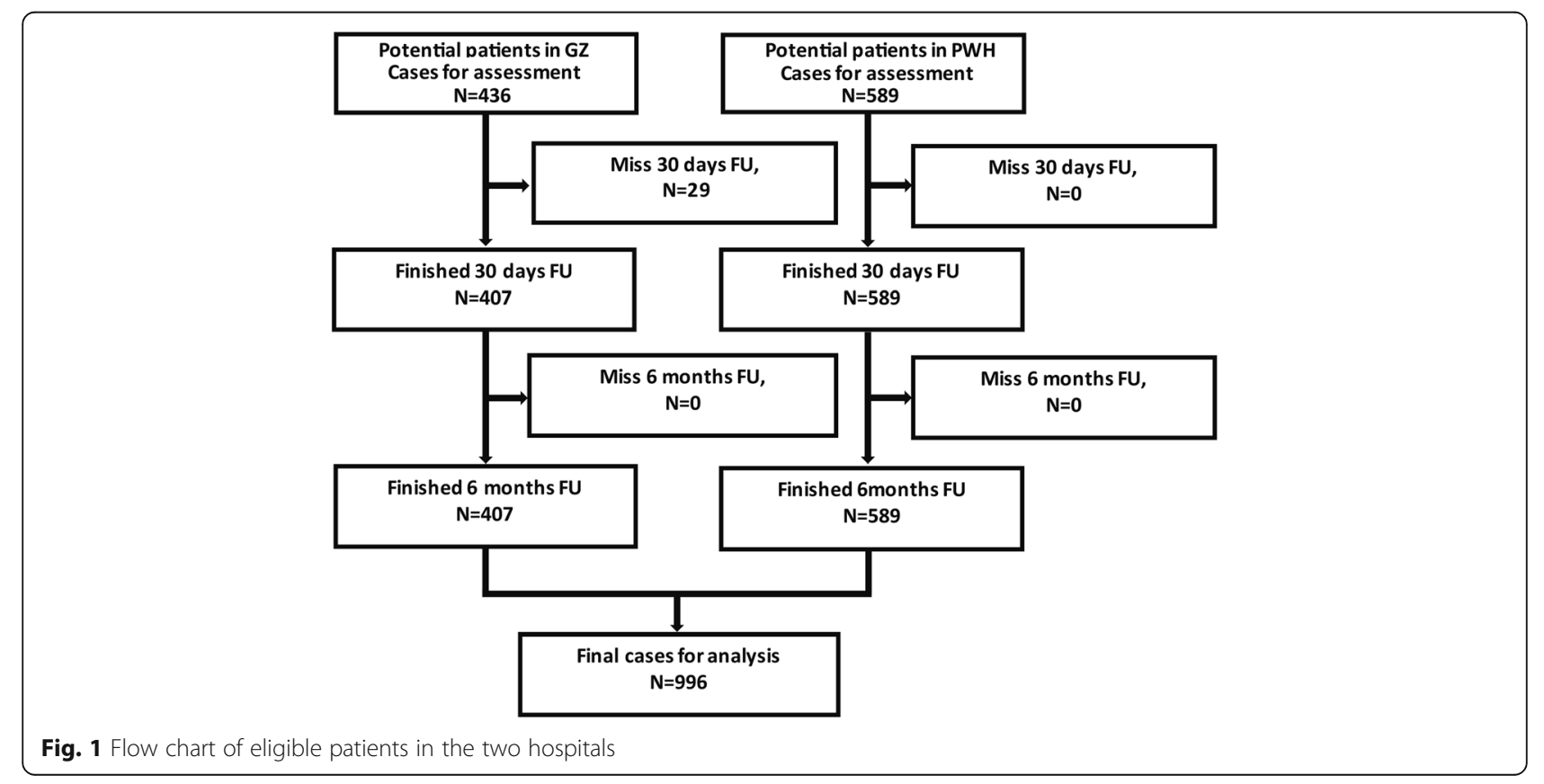


Table 1 Comparison of patient characteristics between the two hospitals ( $N=996)$

\begin{tabular}{|c|c|c|c|}
\hline & AHGZMU $(n=407)$ & $\mathrm{PWH}(n=589)$ & $P$ \\
\hline Age, years, mean (SD) & $66.6(14.4)$ & $63.7(14.6)$ & $0.002^{*}$ \\
\hline Males, $n(\%)$ & $220(54.1)$ & $325(55.2)$ & 0.726 \\
\hline \multicolumn{4}{|l|}{ Past histories } \\
\hline Smoker, $n(\%)$ & $69(17.0)$ & $78(13.2)$ & 0.105 \\
\hline Hypertension, n (\%) & $245(60.2)$ & $362(60.9)$ & 0.688 \\
\hline Diabetes, n (\%) & 79 (19.4) & $153(26.0)$ & $0.016^{*}$ \\
\hline Hyperlipidemia, $n$ (\%) & $92(22.6)$ & $266(45.2)$ & $<0.0001^{*}$ \\
\hline Chronic heart failure, $n(\%)$ & $26(6.4)$ & $62(10.5)$ & $0.024^{*}$ \\
\hline Family heart disease, $n(\%)$ & $72(17.7)$ & $125(21.2)$ & 0.169 \\
\hline Stroke, $n(\%)$ & $26(6.4)$ & $72(12.2)$ & $0.002^{*}$ \\
\hline Peripheral arterial disease, $n$ (\%) & $6(1.5)$ & $20(3.4)$ & 0.062 \\
\hline \multicolumn{4}{|l|}{ NHYA classification } \\
\hline NHYA class I, n (\%) & $234(57.5)$ & $401(68.1)$ & $0.001^{*}$ \\
\hline NHYA class II, n (\%) & $118(29.0)$ & $75(12.7)$ & $<0.0001$ \\
\hline NHYA class III, n (\%) & $51(12.5)$ & $108(18.3)$ & $0.014^{*}$ \\
\hline NHYA class IV, n (\%) & $4(1.0)$ & $5(0.8)$ & 0.826 \\
\hline \multicolumn{4}{|l|}{ First ED characteristics } \\
\hline Heart rate, bpm, mean (SD) & $88.4(27.8)$ & $82.0(19.1)$ & $<0.0001^{*}$ \\
\hline Systolic blood pressure, mmHg, mean (SD) & $141.3(30.1)$ & $148.7(25.9)$ & $0.001^{*}$ \\
\hline Diastolic blood pressure, mmHg, mean (SD) & $78.2(17.0)$ & $80.1(15.4)$ & 0.018 \\
\hline \multicolumn{4}{|l|}{ First lab results in ED } \\
\hline Creatinine, $\mu \mathrm{moL} / \mathrm{L}$, median (IQR) & $88.0(71.0,109.0)$ & $81.0(66.0,97.0)$ & $<0.0001^{*}$ \\
\hline \multicolumn{4}{|l|}{ Troponin } \\
\hline$\leq 1 \times$ & $224(78.9)$ & $420(72.4)$ & $<0.0001^{*}$ \\
\hline $1 x-2 x$ & $21(7.4)$ & $85(14.7)$ & $<0.0001^{*}$ \\
\hline $2 x-3 x$ & $8(2.8)$ & $22(3.8)$ & 0.108 \\
\hline$\geq 3 x$ & $31(10.9)$ & $53(9.1)$ & 0.441 \\
\hline Number of CK done, $n(\%)$ & $399(98.0)$ & $339(57.6)$ & $<0.0001^{*}$ \\
\hline Number of CK-MB done, $n(\%)$ & $399(98.0)$ & $0(0)$ & $<0.0001^{*}$ \\
\hline ST segment depression, $n(\%)$ & $80(8.0)$ & $19(1.9)$ & $<0.0001^{*}$ \\
\hline Onset- ED time,hour, median (IQR) & $4.03(1.5-13.21)$ & $6.52(2.01-23.27)$ & $<0.0001^{*}$ \\
\hline ED-ECG time, hour, median (IQR) & $0.17(0.03-0.47)$ & $0.18(0.12-0.28)$ & 0.234 \\
\hline HEART Score, median (IQR) & $4(3,6)$ & $4(2,5)$ & $<0.0001^{*}$ \\
\hline Number of admission & $231(56.8)$ & $334(56.7)$ & 0.987 \\
\hline \multicolumn{4}{|l|}{ Final diagnoses } \\
\hline Ischemia coronary chest pain & $120(29.5)$ & $163(27.7)$ & 0.534 \\
\hline NSTEMI, $n(\%)$ & $40(9.8)$ & $32(5.4)$ & \\
\hline Unstable angina, $n$ (\%) & $65(16.0)$ & $71(12.1)$ & \\
\hline Stable angina, $n(\%)$ & $15(3.7)$ & $60(10.2)$ & \\
\hline Non-ischaemia cardiac chest pain, $n(\%)$ & $136(33.4)$ & $84(14.3)$ & $<0.0001^{*}$ \\
\hline Non-cardiac chest pain, $n(\%)$ & $151(37.1)$ & $342(58.1)$ & $<0.0001^{*}$ \\
\hline
\end{tabular}


Table 2 Comparison of 30-day and 6-month mortality in patients with different diagnoses between the two hospitals ( $N=996)$

\begin{tabular}{|c|c|c|c|c|c|c|}
\hline & $\begin{array}{l}\text { 30-day mortality in GZ } \\
(n=407)\end{array}$ & $\begin{array}{l}\text { 30-day mortality in HK } \\
(n=589)\end{array}$ & $P$ & $\begin{array}{l}\text { 6-month mortality in GZ } \\
(n=407)\end{array}$ & $\begin{array}{l}\text { 6-month mortality in HK } \\
(n=589)\end{array}$ & $P$ \\
\hline \multicolumn{7}{|l|}{ Final diagnoses } \\
\hline $\begin{array}{l}\text { Ischemia coronary chest } \\
\text { pain }\end{array}$ & $6(1.5)$ & $0(0)$ & $0.005^{*}$ & $7(1.7)$ & $2(0.3)$ & $0.036^{*}$ \\
\hline NSTEMI, $n$ (\%) & $4(1.0)$ & $0(0)$ & & $5(1.2)$ & $1(0.2)$ & \\
\hline Unstable angina, n (\%) & $2(0.5)$ & $0(0)$ & & $2(0.5)$ & $0(0)$ & \\
\hline Stable angina, $n(\%)$ & $0(0)$ & $0(0)$ & & $0(0)$ & $1(0.2)$ & \\
\hline $\begin{array}{l}\text { Non-ischaemia cardiac } \\
\text { causes, } n(\%)\end{array}$ & $4(1.0)$ & $0(0)$ & 0.28 & $5(1.2)$ & $3(0.5)$ & 0.283 \\
\hline Non-cardiac causes, $n$ (\%) & $5(1.2)$ & $2(0.3)$ & 0.129 & $7(1.7)$ & $6(1.0)$ & 0.339 \\
\hline Total & $15(3.7)$ & $2(0.3)$ & $<0.0001^{*}$ & $19(4.7)$ & $11(1.9)$ & 0.011 \\
\hline
\end{tabular}

The death reasons of non-cardiac chest pain were: pulmonary embolism $=1$ in GZ, pneumonia $=1$ in $\mathrm{GZ}$ and 3 in $\mathrm{HK}$, ischaemic stroke $=1$ in $\mathrm{GZ}$, cancer $=1$ in $\mathrm{GZ}$ and 3 in $\mathrm{HK}$, other reasons $=3$ in $\mathrm{GZ}$

The death reasons of non-ischaemic cardiac chest pain were: heart failure $=2$ in $\mathrm{GZ}$ and 2 in HK, Valvular heart disease $=1$ in $\mathrm{HK}, \mathrm{Aortic}$ dissection $=3$ in $\mathrm{GZ}$ *statistically significant

non-ischaemic cardiac and non-cardiac causes in GZwere $1.5 \%, 1.0 \%$ and $1.7 \%$ respectively. No one died of ischaemia and non-ischaemia cardiac aetiologies in HK up to 30-days follow-up. The 30-day mortality rates for non-cardiac causes was $0.7 \%$ in $\mathrm{HK}$. The 6-month mortality rates of ischaemic chest painin GZ were statistically higher than those in HK.

The 6-month mortality of ischemia, non-ischaemic cardiac and non-cardiac causes in GZ were $1.7 \%, 1.2 \%$ and $1.7 \%$ respectively, whilst in HK these were $0.3 \%, 0.5 \%$ and $1.0 \%$ respectively. NSTEMI patients demonstrated a higher mortality than other ischaemic chest pain patients at 30-day and 6-month follow-up. Compared with cardiac aetiologies, higher mortality was observed for non-cardiac causes. Most diagnoses of non-cardiac chest pain were pulmonary embolism, pneumonia, ischemia stroke and cancer (see Table 2 for details).

\section{Univariate and multivariate logistic regression for 30-day and 6-month mortality}

Table 3 shows patients were more likely to die after 30 days if they were older, had ST-segment deviation, a history of worse NHYA class and lower SBP and DBP, higher serum creatinine and troponin level at ED admission, and a higher HEART score. After multivariate logistic regression, serum creatinine level was the only independent predictor of 30-day mortality.

Table 4 shows patients were more likely to die by 6 months if they were older, had ST-segment depression, a history of chronic heart failure, hyperlipidemia and worse NHYA class, lower SBP, higher serum creatinine and troponin level at ED admission, and higher HEART score. After multivariate logistic regression, serum creatinine level and SBP were the only independent predictors of 6-month mortality.

\section{Survival analysis in two hospitals}

Figure 2 showed the corresponding Kaplan-Meier eventfree survival curves for different hospitals. Kaplan-Meier analysis demonstrated a significantly increased probability of death ofchest pain patients in GZ after 30 days and 6 months $(P<0.0001$ for 30 -day mortality using the log-rank test, and $P=0.010$ for 6 -month mortality using the log-rank test).

In Cox regression analysis, the unadjusted HR for 30-day $(\mathrm{HR}=11.05,95 \% \mathrm{CI} 2.53$ to $48.32, P=0.001)$ and 6-month (HR $=2.55,95 \%$ CI 1.22 to $5.37, P=0.013$ ) mortality in GZ was significantly higher (Table 5). After adjusting for age, SBP, ST segment depression, NHYA classification, troponin, HEART score and serum creatinine, the adjusted HR for 30-day ( $\mathrm{HR}=8.98,95 \% \mathrm{CI} 1.78$ to $45.2, P=0.008)$ mortality in GZ was still significantly higher.

After adjusting for age, hyperlipidemia, CHF, SBP, ST segment depression, NHYA classification, troponin, HEART score and serum creatinine, the adjusted HR for 6-month $(\mathrm{HR}=1.77,95 \% \mathrm{CI} 0.73$ to $4.26, P=0.205)$ mortality in GZ was not significantly higher than that in $\mathrm{HK}$.

\section{Discussion}

This is the first prospective cohort study to compare 30day and 6-month mortality of suspected cardiac chest pain patients admitted to EDs between two teaching hospitals located in two different cities in southern China with similar ethnicity but differenthealthcare systems [11]. The chest pain patients in GZ had a higher 30-day and 6-month mortalitythan in HK. The findings suggest that the different outcomes were associated with the varying clinical characteristics of patients and different distributions of diagnoses under two differenthealthcare systems. GZ has integrated many features of healthcare system associated with market economies, while its 
Table 3 Univariate and multivariate logistic regression for 30-day mortality $(N=996)$

\begin{tabular}{|c|c|c|c|c|}
\hline & Unadjusted Odds Ratio (95\% Cl) & $P$ & Adjusted Odds Ratio $(95 \% \mathrm{Cl})$ & $P$ \\
\hline Age, years & $1.069(1.023-1.117)$ & $0.003^{*}$ & $1.031(0.977-1.089)$ & 0.262 \\
\hline Males (vs Females) & $2.008(0.702-5.744)$ & 0.193 & & \\
\hline \multicolumn{5}{|l|}{ Past histories } \\
\hline Smoker (vs non smoker) & $2.256(0.644-7.905)$ & 0.204 & & \\
\hline Hypertension (vs non hypertension) & $1.094(0.413-2.899)$ & 0.857 & & \\
\hline Diabetes (vs non diabetes) & $0.702(0.2-2.464)$ & 0.58 & & \\
\hline \multicolumn{5}{|l|}{ Hyperlipidemia (vs non hyperlipidemia) } \\
\hline CHF (vs non CHF) & $2.254(0.635-7.998)$ & 0.209 & & \\
\hline FHD (vs non FHD) & $1.153(0.328-4.053)$ & 0.824 & & \\
\hline Stroke (vs non stroke) & $0.568(0.075-4.332)$ & 0.586 & & \\
\hline \multicolumn{5}{|l|}{ PAD (vs non PAD) } \\
\hline \multicolumn{5}{|l|}{ First ED characteristics } \\
\hline Heart rate & $1.003(0.982-1.024)$ & 0.804 & & \\
\hline SBP & $0.980(0.962-0.990)$ & $0.044^{*}$ & $0.990(0.963-1.089)$ & 0.486 \\
\hline DBP & $0.973(0.942-1.005)$ & 0.1 & $1.02(0.97-1.072)$ & 0.435 \\
\hline NHYA class III, IV (vs NHYA class I,II) & $3.557(1.334-9.481)$ & $0.011^{*}$ & $1.554(0.405-5.972)$ & 0.521 \\
\hline Troponin Elevation $\geq 2 \times(v s<2 \times)$ & $10.385(1.228-87.874)$ & $0.032^{*}$ & $2.471(0.613-9.955)$ & 0.203 \\
\hline Creatinine elevation (vs normal) & $11.643(3.947-34.347)$ & $<0.0001^{*}$ & 9.189 (2.319-36.407) & $0.002^{*}$ \\
\hline ST segment depression (vs normal) & $0.175(0.061-0.5)$ & $0.001^{*}$ & $1.026(0.973-1.083)$ & 0.34 \\
\hline HEART Score & $1.622(1.228-2.143)$ & $0.001^{*}$ & $0.706(0.404-1.231)$ & 0.22 \\
\hline Onset- ED time & $0.966(0.919-1.015)$ & 0.172 & & \\
\hline ED-ECG time & $1(1-1)$ & 0.949 & & \\
\hline
\end{tabular}

CHF Chronic heart failure, FHD Family history of heart disease PAD Peripheral arterial disease, SBP Systolic blood pressure, DBP Diastolic blood pressure Adjusted for age, SBP, DBP,ST segment depression, NHYA classification, troponin, HEART score and serum creatinine

${ }^{*}$ statistically significant

overall economy is largely centrally planned. In contrast,Hong Kong adopt thehealthcare system are come from England and organizational health systems that are commonly seen in centrally planned economies, while its economy functions as a highly capitalisticenterprise $[10,13]$.

\section{Different distributions of chest pain patients between two hospitals}

Over $50 \%$ of patients were diagnosed as non-cardiac chest pain in HK, a much higher rate than in GZ (37.1\%). This may reflect the different healthcare systems. In Guangzhou, chest pain patients at low risk often present to clinics instead of EDs during office hours. The ED is the only choice for patients with chest pain in HK due to lower medical costs. So, the patients with urgent and severe chest pain/symptoms would likely to present to EDs in GZ. Compared to other studies, the proportion of non-cardiac chest pain patientsin this study was low. The proportions in GZ and HK were $50 \%$ and $37.1 \%$ respectively compared to $67.2 \%-70 \%$ in other studies [14-16]. A lower proportion of non-cardiac chest pain patients may allow earlier discharge from the ED and place less stress on ED and hospital services.
There were no significant differences in 30-day and 6month mortality for non-ischemic cardiac chest pain and non-cardiac chest pain between the two hospitals. However, there was significantly higher 30-day and 6month mortality rates for ischemic cardiac chest pain patients in GZ. ACS is a high-risk disease with significant mortality [9, 17]. The current study excluded STEMI patients but NSTEMI and UA patients were recruited. The combined proportion of NSTEMI and UA in GZ was $25.8 \%$, which was higher than thatin HK (17.5\%). The proportions of NSTEMI and UA were also higher than those in Cullen's study (8.7\%) whilst non ACS cardiovascular-related conditions were 20.8\% [14].

\section{Different clinical characteristics and outcomes of patients between two hospitals}

Since the patients with severe chest pain/symptoms would likely to present to EDs in GZ while those patients with mild chest pains/symptoms would visit clinics, so the baseline clinical characteristicsof patients in GZ were worse than those in HK. The patients were older, had lower proportion of NHYA class I and higher creatinine level, higherproportion of ST-segment depression, lower 
Table 4 Univariate and multivariate logistic regression for 6-month mortality ( $N=996)$

\begin{tabular}{|c|c|c|c|c|}
\hline & Unadjusted Odds Ratio (95\% Cl) & $P$ & Adjusted Odds Ratio (95\% Cl) & $P$ \\
\hline Age, years & $1.074(1.038-1.112)$ & $<0.0001^{*}$ & $1.033(0.98-1.088)$ & 0.233 \\
\hline Males (vs Females) & $0.508(0.230-1.121)$ & 0.093 & $0.592(0.17-2.053)$ & 0.592 \\
\hline \multicolumn{5}{|l|}{ Past histories } \\
\hline Smoker (vs non smoker) & $0.755(0.332-1.715)$ & 0.502 & & \\
\hline Hypertension (vs non hypertension) & $1.291(0.598-2.789)$ & 0.515 & & \\
\hline Diabetes (vs non diabetes) & $1.002(0.425-2.367)$ & 0.996 & & \\
\hline Hyperlipidemia (vs non hyperlipidemia) & $0.191(0.058-0.635)$ & $0.007^{*}$ & $0.00(0.00-0.00)$ & 0.996 \\
\hline CHF (vs non CHF) & $4.812(2.132-10.861)$ & $<0.0001^{*}$ & $3.454(0.705-16.93)$ & 0.126 \\
\hline FHD, (vs non FHD) & $0.442(0.133-1.473)$ & 0.184 & & \\
\hline Stroke (vs non stroke) & $1.019(0.303-1.473)$ & 0.976 & & \\
\hline PAD (vs non PAD) & $1.298(.170-9.909)$ & 0.801 & & \\
\hline \multicolumn{5}{|l|}{ First ED characteristics } \\
\hline Heart rate & $1.007(0.993-1.022)$ & 0.318 & & \\
\hline SBP & $0.979(0.965-0.993)$ & $0.004^{*}$ & $0.974(0.95-0.999)$ & $0.045^{*}$ \\
\hline DBP & $0.977(0.954-1.001)$ & 0.061 & $1.045(0.998-1.094)$ & 0.114 \\
\hline NHYA class III, IV (vs NHYA class I,II) & $5.314(2.545-11.095)$ & $<0.0001^{*}$ & $2.519(0.685-9.266)$ & 0.165 \\
\hline Troponin Elevation $\geq 2 x(v s<2 x)$ & $8.04(1.693-38.185)$ & $0.009^{*}$ & $2.983(0.494-18.01)$ & 0.233 \\
\hline Creatinine elevation (vs normal) & $11.137(4.23-29.326)$ & $<0.0001^{*}$ & $7.48(2.042-27.369)$ & $0.002^{*}$ \\
\hline ST segment depression (vs normal) & $4.190(1.968-8.917)$ & $<0.0001^{*}$ & $1.006(0.954-1.061)$ & 0.815 \\
\hline HEART Score & $1.603(1.310-2.027)$ & $<0.0001^{*}$ & $0.81(0.487-1.349)$ & 0.418 \\
\hline Onset- ED time & $0.986(0.964-1.008)$ & 0.216 & & \\
\hline ED-ECG time & $1.00(1.000-1.000)$ & 0.922 & & \\
\hline
\end{tabular}

CHF Chronic heart failure, FHD Family history of heart disease, $P A D$ Peripheral arterial disease, SBP Systolic blood pressure, $D B P$ Diastolic blood pressure Adjusted for age, gender, hyperlipidemia, CHF, SBP, DBP, ST segment depression, NHYA classification, troponin, HEART score and serum creatinine ${ }^{*}$ statistically significant

proportion of negative TnT and higher HEART scoreat ED presentation. Those factors have also been shown to be associated with 30-day and 6-month mortality. The same parameters have been verified to predict short- and long-term risks of death from ACS [18-24]. Compared to HK, lower proportions of patients with risk factors were seen in GZ, whilst more patients with worse NHYA class were observed. This suggests that many chest pain patients in GZ did not have any knowledge of cardiac risk factors and were unaware of the presence of those risk factors, therefore the impact of those risk factors might be underestimated. Early detection and good control of risk factors for suspected cardiac chest pain patients may reduce mortality.

Furthermore, elevated serum creatinine level was an independent predictor for 30-day and 6-month mortality in our study.It has also been reported to be associated with worse outcomes for ACS and critically ill patients $[18,23]$. Elevated serum creatinine can reflect vascular damage, renal impairment, endothelial dysfunction and impaired myocardial blood flow [24-27]. A multinational registry study by Tang et al. showed that SBP could predict the 6-month mortality of all subsets of ACS. As SBP increases by $10 \mathrm{mmHg}$, the hazard ratio would decrease by 0.95 [27].

Several studies have reported that the HEART score is not only related to the severity of chest pain in EDs but also predicts the occurrence of clinical endpoints [2832]. Our study also demonstrated that the HEART score was positively associated with 30-day and 6-month mortality of suspected cardiac chest pain patients in the ED setting.

The dissimilarity of healthcare systems in the two cities is another reason for the different ED management. Limited capacity of hospital emergency care services, high out-of-pocket expenses with the need for up-front payment, prolonged discussions with the patient and families for both obtaining consent and pooling funds are likely to be major contributing factors for the long prehospital, in-hosptial delay and refused some expensive treatments [13,32].

In ED chest pain pathways, guidelines recommend that an ECG should be recorded within $10 \mathrm{~min}$ and myocardial injury biomarkers should be measured as soon as possible in all suspected cardiac chest pain patients $[4,9]$. The ED-ECG time and the proportion of 


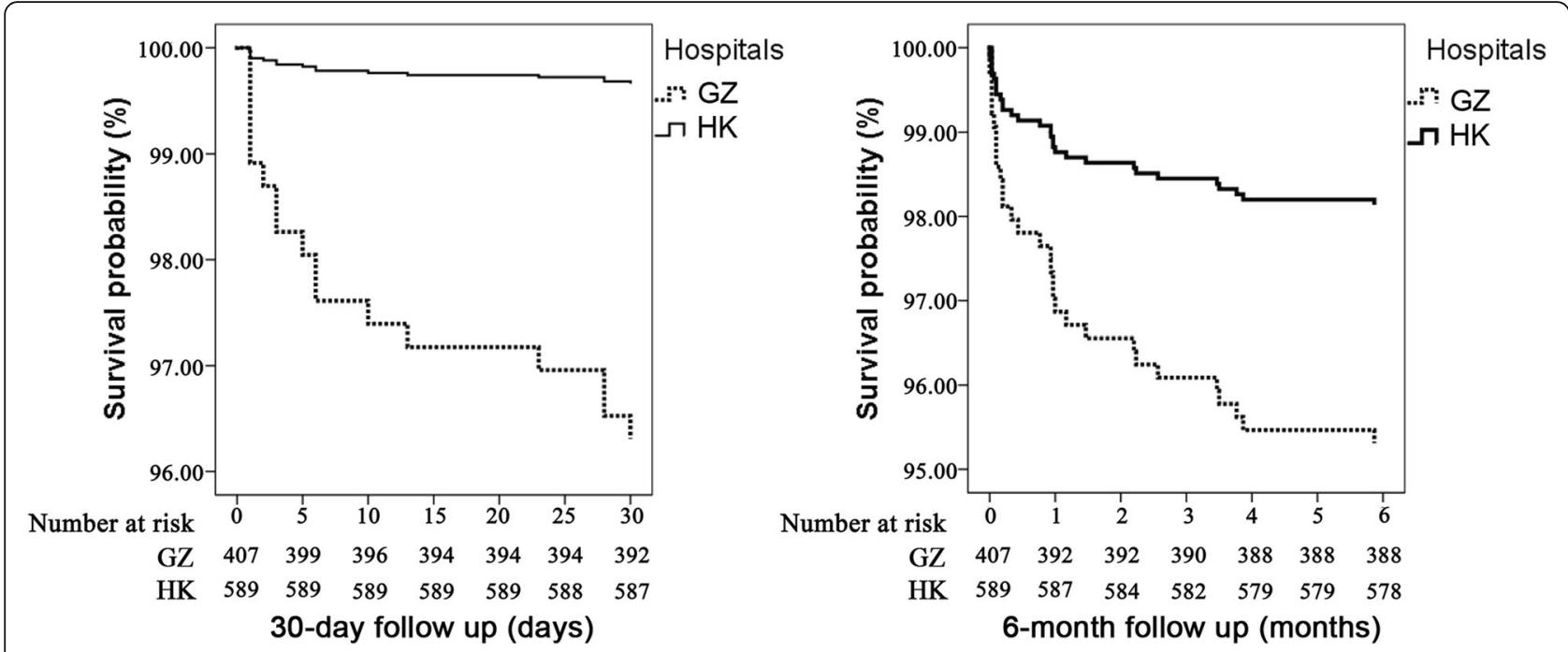

Fig. 2 Kaplan-Meier event-free survival curves in GZ and HK. Kaplan-Meier analysis demonstrated a significant increased probability of mortality during 30-day (Log Rank $P<0.0001$ ) and 6-month follow-up in GZ (Log Rank $P=0.010$ )

patients having myocardial injury biomarkers measured in both hospitals met these guidelines.Myocardial injury biomarkers, such as troponin, CK and CK-MB are used to identify myocardial injury [33]. Many studies have shown that troponinis superior to $\mathrm{CK}$ and CK-MB as a biomarker for detection of myocardial injury [19, 20]. Therefore, troponin testing is recommended in most guidelines for its high sensitivity and specificity [17]. However in GZ, CK and CK-MB are still mainly used for the diagnosis of AMI and ACS due to their lower costs. High expenses with the need for up-front payment of troponin testing are likely to be a major contributing factor for the lower rate of use of troponin testing in GZ. The lower sensitivities and specificities of CK and CKMB might contribute to the delayed diagnoses of AMI/ ACS and later treatment of patients, possibly contributing to the higher early mortality in GZ. Except the mortaliy, readmissions after ACS are associated with higher

Table 5 Unadjusted and adjusted hazard ratios of all-cause mortality at 30-days and 6-months in the two hospitals

\begin{tabular}{|c|c|c|c|c|}
\hline & $\mathrm{HR}(95 \% \mathrm{Cl})$ & $P$ & Adjusted HR (95\% Cl) & $P$ \\
\hline \multicolumn{5}{|c|}{ 30-day all-cause mortality } \\
\hline $\mathrm{GZ}$ & $11.05(2.53-48.32)$ & $0.001^{*}$ & $8.98(1.78-45.2)$ & $0.008^{*}$ \\
\hline HK & Reference & & Reference & \\
\hline \multicolumn{5}{|c|}{ 6-month all-cause mortality } \\
\hline GZ & $2.55(1.22-5.37)$ & $0.013^{*}$ & $1.77(0.73-4.26)$ & 0.205 \\
\hline HK & Reference & & Reference & \\
\hline
\end{tabular}

30-day mortality was adjusted for age, SBP, DBP, ST segment depression, NHYA classification, troponin, HEART score and serum creatinine 6-month mortality was adjusted for age, gender, hyperlipidemia, CHF, SBP, DBP, ST segment depression, NHYA classification, troponin, HEART score and serum creatinine

*statistically significant long-term all-cause mortality [34].However, our study did not show the significant difference of 30-day and 6-month readmission rate in in HK and GZ. The reason was that the patients we enrolled were suspected cardiac chest pain patients instead of ACS.

\section{Strengths and limitations}

The strengths of this study include the fact it is the first comparison of outcomes in different healthcare systems in a similarly populated region of southern China, which effectively eliminates many confounding factors in any comparison. The current study also has some limitations, including the sample size of this study which met our precalculated size but may still be considered small by international standards. Secondly, patient risk factors (i.e. the past medical histories) may have been underestimated due to variations in history taking between the two centres. Thirdly, as a prospective observational study, propensity score with matching would attempt to reduce the bias due to confounding variables. However, this method requires large sample size. Also, the procedure only controls for observed variables, so any hidden bias due to latent variables may remain after matching. Logistic regression and Cox regression can meet the need of this study.

\section{Conclusion}

The 30-day and 6-month mortality of patients with suspected cardiac chest pain in Guangzhou were higher than in Hong Kong due to different baseline clinical characteristics of patients at ED presentation and different distributions of diagnoses which were associated with different healthcare systems. Serum creatinine and SBP were independent predictors of 30-day and 6-month 
mortality. Improved patient knowledge of risk factors along with early detection and good control of risk factors may lead to better outcomes for cardiac chest pain patients in southern China.

\section{Additional file}

Additional file 1: Definition of HEART score [29]: There were five elements: history, ECG, age,risk factors and troponin in HEART score. Each variable was scored as 0,1 or 2 points. Each patient will receive a score of 1-10. (DOCX $32 \mathrm{~kb})$

\section{Abbreviations}

ACS: Acute coronary syndrome; AHGZMU: The Second Affiliated Hospital of Guangzhou Medical University; CABG: Coronary artery bypass grafting; CAD: Coronary artery disease; CK: Creatine Kinase; CK-MB: Creatine Kinase Isoenzyme-MB; CMS: Clinical Management System; CTnT: Cardiac troponin T; ED: Emergency department; GZ: Guangzhou; HK: HongKong; IQR: Interquartile range; MACE: Major adverse cardiac events; NSTEMI: Non ST-segment elevation myocardial infarction; PPCI: Primary percutaneous coronary intervention; PWH: Prince of Wales Hospital; STEMI: ST elevation myocardial infarction; US: United States

\section{Funding}

The Bureau of Science and Information Technology of Guangzhou Municipality (Grant No.2013 J4500009).

The Scientific Research Project of the Guangzhou Education Bureau (Grant No. 1201610645).

The Key Medical Disciplines and Specialties Program of Guangzhou

\section{Availability of data and materials}

The datasets used and/or analysed during the current study are available from the corresponding author on reasonable request.

\section{Authors' contributions}

Conceived and designed the study: CAG, JHL \&THR. Monitored the entire planning, execution, and analysis of the study: CAG, JHL \&THR. Obtained the ethical approval and grant for the study: THR, CXH \& JHL. Participated in staff training in the application of the scores, recruitment of participants and data collection for the study: CAG, THR, CXH, JHL, MJR, LPY \& LYM. Analyzed the data and performed the statistical analysis: CAG, JHL, LYM \& MJR. Provided advice on the study methods and manuscript writing: CAG, THR, JHL, KH \& $\mathrm{CXH}$. Wrote the first draft of the manuscript and prepared the manuscript: CAG \& JHL. Contributed to the final version: All authors read and approved the final manuscript

\section{Ethics approval and consent to participate}

Ethical approval was obtained from the joint Chinese University of Hong Kong-New Territories East Cluster (CUHK-NTEC) Clinical Research Ethics Committee in Hong Kong and the Institutional Review Board in Guangzhou to conduct a prospective observational study in patients with chest pain. Written informed consent was obtained from each patient after a verbal and written explanation of the study was provided. If patients were unable to give signed consent, written consent would obtained from relatives on behalf of patients. Patients were informed that they could withdraw from the study at any time.

\section{Competing interests}

The authors declare that they have no competing interests.

\section{Publisher's Note}

Springer Nature remains neutral with regard to jurisdictional claims in published maps and institutional affiliations.

\section{Author details}

'Emergency Department, The Second Affiliated Hospital of Guangzhou Medical University, Guangzhou, China. ${ }^{2}$ Accident and Emergency Medicine
Academic Unit, Chinese University of Hong Kong, Prince of Wales Hospital, Main Clinical Block and Trauma Centre, Shatin, N.T, Hong Kong, China.

Received: 18 December 2017 Accepted: 20 April 2018

Published online: 16 May 2018

References

1. Amsterdam EA, Kirk JD, Bluemke DA, et al. American heart AssociationExercise, cardiac rehabilitation, and prevention Committee of the Council onClinical cardiology, council on cardiovascular nursing, and InterdisciplinaryCouncil on quality of care and outcomes research. Testing of low-risk patientspresenting to the emergency department with chest pain: a scientific statementfrom the American Heart Association. Circulation. 2010;122(17):1756-76. https://doi.org/10.1161/CIR.0b013e3181ec61df.

2. Liu Y, Zhang B, Fu W, et al. A preliminary epidemiological study of the patient population visiting an urban ED in the republic of China. Am J Emerg Med. 1994;12(2):247-9.

3. Bayley MD, Schwartz JS, Shofer FS, Weiner M, Sites FD, Traber KB. The financial burden of emergency department congestion and hospital crowding for chest pain patients awaiting admission. Ann Emerg Med. 2005;45(2):110-7. https://doi.org/10.1016/j.annemergmed.2004.09.010.

4. Hu DY, Zhao MZ. Early recognition and treatment for patients with chest pain. Chin J Gen Pract. 2003;2(2):75-6. https://doi.org/10.3760/cma.j.issn. 1671-7368.2003.02.005

5. Anderson JL, Adams CD, Antman EM, et al. 2012 ACCF/AHA focused update incorporated into theACCF/AHA 2007 guidelines for the management ofpatients with unstable angina/non-ST-elevationmyocardial infarction: a report of the AmericanCollege of Cardiology Foundation/American HeartAssociation Task Force on Practice Guidelines. J AmCollCardiol. 2013; 61(23):e179-347. https://doi.org/10.1016/j.jacc.2013.01.014.

6. Kong X, Yang Y, Gao J, et al. Overview of the health care system in Hong Kong and its referential significance to mainland China. J Chin Med Assoc. 2015;78(10):569-73. https://doi.org/10.1016/j.jcma.2015.02.006

7. Graham CA, Chan JW, Chan CP, Cattermole GN, Rainer TH. Prospective validation of thrombolysis in myocardial infarction and front door thrombolysis in myocardial infarction risk scores in Chinese patients presenting to the ED with chest pain. Am J Emerg Med. 2014;32(11):133944. https://doi.org/10.1016/j.ajem.2014.08.032.

8. Rainer $\mathrm{TH}$, Leung $\mathrm{YK}$, Lee $\mathrm{A}$, et al. Add-on tests for improving riskstratification in emergency department patients with chest pain who are at low to moderate risk of 30-day major adverse cardiac events. Int J Cardiol. 2016;220:299-306. https://doi.org/10.1016/j.jijcard.2016.05.057.

9. Huo Y. Promoting chest pain center accreditation is an essential way to improve the management of acute myocardial infarction in China. Zhonghua Xin Xue Guan Bing Za Zhi. 2014;42(8):637-8. https://doi.org/10. 3760/cma.j.jissn.0253-3758.2014.08.004.

10. Fitzner KA, Coughlin S, Tomori C, Bennett CL. Health care in Hong Kong and mainland China: one country, two systems? Health Policy. 2000,53:147-55.

11. Chen X, Li M, Jiang H, et al. STEMl Outcomes in Guangzhou and Hong Kong: Two-Centre Retrospective Interregional Study. PLoS One. 2016;11(3): e0149981. https://doi.org/10.1371/journal.pone.0149981.

12. Kip KE, Hollabaugh K, Marroquin OC, Williams DO. The problem with composite end points in cardiovascular studies: the story of major adverse cardiac events and percutaneous coronary intervention. J Am Coll Cardiol. 2008;51(7):701-7. https://doi.org/10.1016/j.jacc.2007.10.034

13. Ma J, Lu M, Quan H.From a national, centrally planned health system to a system based on the market: lessons from China. Health Aff (Millwood). 2008;27(4):937-48. https://doi.org/10.1377/hlthaff.27.4.937.

14. Cullen L, Greenslade J, Merollini K, et al. Cost and outcomes of assessing patients with chest pain in an Australian emergency department. Med J Aust. 2015;202(8):427-32.

15. Soltani M, Mirzaei M, Amin A, et al. Predictors of adverse outcomes of patients with chest pain and primary diagnosis of non-cardiac pain at the time of discharge from emergency department: a 30-days prospective study. Ethiop J Health Sci. 2016;26(4):305-10.

16. Durand E, Delos A, Chaib A, et al. Performance assessment of a chest pain unit: preliminary 2-year experience in the European GeorgesPompidou hospital. Arch Cardiovasc Dis. 2009;102(12):803-9. https://doi.org/10.1016/j. acvd.2009.09.008.

17. EA A, Wenger NK, Brindis RG, et al. 2014 AHA/ACC guideline for the Management of Patients with non-ST-elevation acute coronary syndromes: 
a report of the American College of Cardiology/American Heart Association task force on practice guidelines. J Am Coll Cardiol. 2014;64(24):e139-228. https://doi.org/10.1016/jacc2014.09.017.

18. Eagle KA, Lim MJ, Dabbous OH, et al. A validated prediction model for all forms of acute coronary syndrome: estimatingthe risk of 6-month postdischarge death in an international registry. JAMA. 2004;291(22):272733. https://doi.org/10.1016/jannemergmed2011.07.026.

19. Ooi SB, Lim YT, Lau TC, Chia BL, Pillai S, Liu T. Value oftroponin-T rapid assay, cardiac enzymes, electrocardiogram and history of chest pain in the initial diagnosis of myocardial infarction in the emergency department. Eur J Emerg Med. 2000;7(2):91-8.

20. Johnson PA, Goldman L, Sacks DB, et al. Cardiac troponin T as a marker for myocardial ischemia in patients seen at the emergency department for acute chest pain. Am Heart J. 1999;137(6):1137-44.

21. Alexander KP, Newby LK, Cannon CP, et al. American Heart Association Council on Clinical Cardiology, Society of Geriatric Cardiology.Acute coronary care in the elderly, part I: Non-ST-segment-elevation acute coronary syndromes: a scientific statement for healthcare professionals from the American Heart Association Council on Clinical Cardiology: in collaboration with the Society of Geriatric Cardiology. Circulation. 2007; 115(19):2549-69. https://doi.org/10.1161/CIRCULATIONAHA.107.182616.

22. AT T, Ramos R, Toste A, et al. Impact of age on treatment and outcomes after acute myocardial infarction, particularly in very elderly patients. Rev Port Cardiol. 2011;30:897-903. https://doi.org/10.1016/jrepc201109013.

23. Kaddourah A, Basu RK, Bagshaw SM, Goldstein SL. AWARE InvestigatorsEpidemiology of Acute Kidney Injury in Critically III Children and Young Adults. N Engl J Med. 2017;376(1):11-20. https://doi.org/10.1056/ NEJMoa1611391.

24. Zhao L, Wang L, Zhang Y. Elevated admission serum creatinine predicts poor myocardial blood flow and one-year mortality in ST-segment elevation myocardial infarction patients undergoing primary percutaneous coronary intervention. J Invasive Cardiol. 2009;21:493-8.

25. Mann JF, Gerstein HC, Pogue J, Bosch J, Yusuf S. Renal insufficiency as a predictor of cardiovascular outcomes and the impact of ramipril: the HOPErandomized trial. Ann Intern Med. 2001;134:629-36.

26. Sarnak MJ, Levey AS, Schoolwerth AC, et al. American Heart Association councils on kidney in cardiovascular disease, high blood pressure research, clinical cardiology, and epidemiology and Prevention. Kidney disease as a risk factor for development of cardiovascular disease: astatement from the American Heart Association councils on kidney in Cardiovascular disease, high blood pressure research, clinical Cardiology, and epidemiology and prevention. Hypertension. 2003;42:1050-65.

27. Tang EW, Wong CK, Herbison P. Global Registry of Acute Coronary Events (GRACE) hospital discharge risk score accurately predicts long-term mortality post acute coronary syndrome. Am Heart J. 2007;153(1):29-35. https://doi. org/10.1016/j.ahj.2006.10.004.

28. Backus BE, Six AJ, Doevendans PA, Kelder JC, Steyerberg EW, Vergouwe Y. Prognostic factors in chest pain patients: a quantitative analysis of the HEART score. Crit Pathw Cardiol. 2016;15(2):50-5. https://doi.org/10.1097/ HPC.0000000000000075.

29. Backus BE, Six AJ, Kelder JC, et al. A prospective validation of the HEART score for chest pain patients at the emergency department. Int J Cardiol. 2013;168(3):2153-8. https://doi.org/10.1016/j.jicard.2013.01.255.

30. Six AJ, Cullen L, Backus BE, et al. The HEART score for the assessment of patients with chest pain in the emergency department: a multinational validation study. Crit Pathw Cardiol. 2013;12(3):121-6. https://doi.org/10. 1097/HPC.0b013e31828b327e.

31. Mahler SA, Riley RF, Hiestand BC, et al. The HEART Pathway Randomized Trial: Identifying Emergency Department Patients With Acute Chest Pain for Early Discharge. Circ Cardiovasc Qual Outcomes. 2015;8(2):195-203. https:// doi.org/10.1161/CIRCOUTCOMES.114.001384

32. Liu YL, Rao KQ, Wu J, Gakidou E. China's health system performance. Lancet. 2008;372:1914-23. https://doi.org/10.1016/S0140-6736(08)61362-8.

33. Collinson PO, Gaze DC, Morris F, Morris B, Price A, Goodacre S. Comparison of biomarker strategies for rapid rule out of myocardial infarction in the emergency department using ACC/ESC diagnostic criteria. Ann Clin Biochem. 2006;43(Pt 4):273-80. https://doi.org/10.1258/000456306777695555.

34. Moretti C, Meynet I, Ascenzo FD, et al. Sixty-day readmission rate after percutaneous coronary intervention: predictors and impact on long-term outcomes. Eur Heart J Qual Care Clin Outcomes. 2015;1:79-84. https://doi. org/10.1093/ehjqcco/qcv001.

\section{Ready to submit your research? Choose BMC and benefit from:}

- fast, convenient online submission

- thorough peer review by experienced researchers in your field

- rapid publication on acceptance

- support for research data, including large and complex data types

- gold Open Access which fosters wider collaboration and increased citations

- maximum visibility for your research: over $100 \mathrm{M}$ website views per year

At BMC, research is always in progress.

Learn more biomedcentral.com/submissions 